Pure \& Appl. Chem., Vo1. 55, No. 10, pp. 1541-1551, 1983. Printed in Great Britain.
$0033-4545 / 83 \$ 3.00+0.00$ Pergamon Press Ltd. () 1983 IUPAC

\title{
INTERACTIONS BETWEEN UNLIKE POLYMERS VERSUS DILUTE SOLUTION PROPERTIES OF COPOLYMERS
}

Takeshi Fukuda and Hiroshi Inagaki

Institute for Chemical Research, Kyoto University, Uji, Kyoto 611, Japan

\begin{abstract}
Solution properties of the copolymers and polymer blends which are under the influence of an unfavorable heteropolymer interaction are described. A body of experimental and computer simulation data indicates that the overall effects of the interaction on the conformational properties of copolymers are strongly dependent on their chain architecture. Typically, block copolymers are surprisingly insensitive to such an interaction. Even among the statistical class of copolymers, the effects are different for different arrangements of comonomer units along the chain. Moreover, the apparent effects of the interaction vary from solvent to solvent. These phenomena are comprehensively interpreted by postulating that the solubility parameter of a copolymer slightly deviates from the composition average of those of the parent polymers, due to the presence of alternating chemical bonds. Based on this idea, the interaction parameter $X_{12}$ between polystyrene (PS) and poly(methyl methacrylate) (PMMA) has been extracted from the viscometric data on the copolymers. The parameter $X_{12}$ in a ternary solution can be fairly accurately determined by the light scattering method which utilizes the optical cancellation among the scattering components. Application of this technique to PS/PMMA systems has disclosed several facts: For a given pair of the polymers, there exists a characteristic concentration $\phi *$ below which $\chi_{12}$ is approximately constant but above which it increases rather sharply. The value of $X_{12}$ below $\phi *$ is dependent on molecular weight, which can be interpreted according to the dilute solution theory. The value of $X_{12}$ above $\phi *$ is a function of concentration only. These dilute and concentrated solution studies offer another two estimates of $\chi_{12}$ between PS and PMMA, which are in favorable agreement with each other and a1so with the copolymer value. These results suggest that solution data could be, at least in certain cases, used to provide quantitative information on polymerpolymer interactions.
\end{abstract}

\section{INTRODUCTION}

In this article, the solution properties of copolymers and polymer blends are described by referring mainly to the work carried out recently in our laboratory. A central question in this research concerns whether and how it is possible to extract, from solution data, certain quantitative information on the interaction between chemically different polymers.

In the first section we describe qualitative differences in the conformational properties between block and statistical copolymers under the influence of an unfavorable heteropolymer interaction. In the second section, some quantitative discussion is made on the architecture and solvent dependency of the hydrodynamic dimensions of copolymers. The last section is concerned with polymer/polymer/solvent ternary solution studies by the light scattering method specially devised to enhance accuracy. The interaction parameters $\chi_{12}$ between polystyrene and poly(methyl methacrylate) deduced from the copolymer and ternary solution studies are compared and discussed.

\section{ARCHITECTURAL DEPENDENCE OF COPOLYMER CONFORMATION}

Copolymers have unique features resulting from the interactions between chemically different monomer units. The overall effects of the interactions should depend not only on molecular weight and composition but also on chain architecture, i.e., mode of comonomer arrangement along the chain. The importance of this last factor is most easily recognized in a solid state. For example, finely controlled multiphase structures are characteristic of block copolymers but not of statistical copolymers or blended polymers (see, e.g., Ref. $1 \& 2$ ).

In solution as well, the extent to which the effects of such interactions manifest themselves should depend on the copolymer architecture. Of the large number of solution studies on 
copolymers (e.g., Ref. 1-15), rather few are concerned specifically with this dependence. Nevertheless, elucidation of the dependence is important not only to understand the solution properties but also to disclose the nature of the interaction between the relevant homopolymers.

Kotaka et al. $(9,16 \& 17)$ were the first to draw attention to the importance of the architectural dependence of the dilute solution properties of copolymers. Specifically, they carried out a systematic study on copolymers of styrene (S) and methyl methacrylate (MMA) with different architecture and found that the second virial coefficient as well as the long-range and short-range interaction parameters are architecturally dependent.

More recently, Monte Carlo calculations worked out by several authors (18-22) disclosed some unique features of the conformational properties of block copolymers. In Fig. 1, we reproduce some of our results $(18 \& 21)$ obtained for symmetrical $A B$ diblock and $B A B$ triblock chains on a simple cubic lattice. On the calculations, segments of the same kind were allowed to occupy the same lattice point, while those of different kinds were not. Thus, the simulated situation would correspond to that of the real chains, with a rather strong, repulsive heterosegment interaction, which are dissolved in a common theta solvent. The figure gives, as a

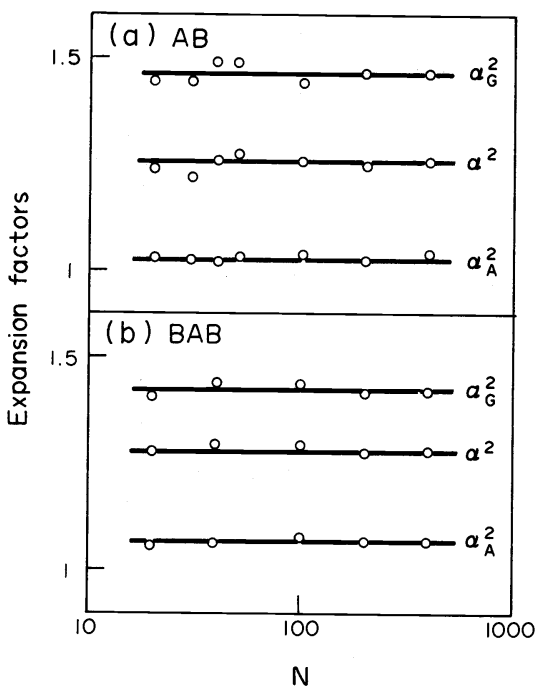

Fig. 1. Plots of expansion factors $\alpha$ vs. chain size $N$ for $A B$ and symmetrical $\mathrm{BAB}$ block copolymers of equimolar composition on a simple cubic lattice with a non-intersecting interaction only between unlike segments (Ref. $18 \& 21$ ).

function of chain size $\mathrm{N}$, the expansion factors relevant to the mean-square radius of the whole chain, $\alpha$, to that of the A block, $\alpha_{A}$, and to the mean-square distance between the centers of mass of the $A$ and (one) B blocks, $\alpha_{G}$ (in the absence of any interaction, $\alpha=\alpha_{A}=$ $\left.\alpha_{G}=1\right)$. Several points may be noteworthy in the figure: In both $A B$ and $B A B$ chains, degrees of expansion are different for different portions of the chains, i.e., $\alpha_{G}>\alpha>\alpha_{A}$. Secondly, $B A B$ chains are slightly more expanded than the $A B$ equivalents. Thirdly, all the $\alpha^{\prime} s$ are apparently independent of $\mathrm{N}$ for sufficiently large $\mathrm{N}$. This last behavior is characteristic of a random-flight chain, but not typical of a chain under the influence of excluded volume effects. These indications were partly confirmed by experimental data (Ref. 18, 21-23).

The most significant implication of the above results may be the remarkable insensitivity of block copolymer conformation to the heterosegment interaction. This by no means indicates that block copolymers assume special conformations such as implied by the terms "intrachain phase separation" and "segregation" (Ref. 8, 24-28). Rather, we would emphasize that the insensitivity is a direct consequence of the architectural features of block copolymers (Ref. 22). Their conformation is "normal" regardless of a repulsive heteroblock interaction.

The insensitivity, in turn, suggests that it is difficult to extract quantitative information on the interaction from their conformational studies (Ref. 22). In contrast, statistical copolymers are characterized by more or less uniform distribution of comonomer units along the chain, and hence a heterosegment interaction should be more directly reflected on the chain conformation. In Fig. 2, we refer to our Monte Carlo result (21) obtained for a statistical copolymer on a tetrahedral lattice. As in the calculations presented in Fig. 1, we imposed 


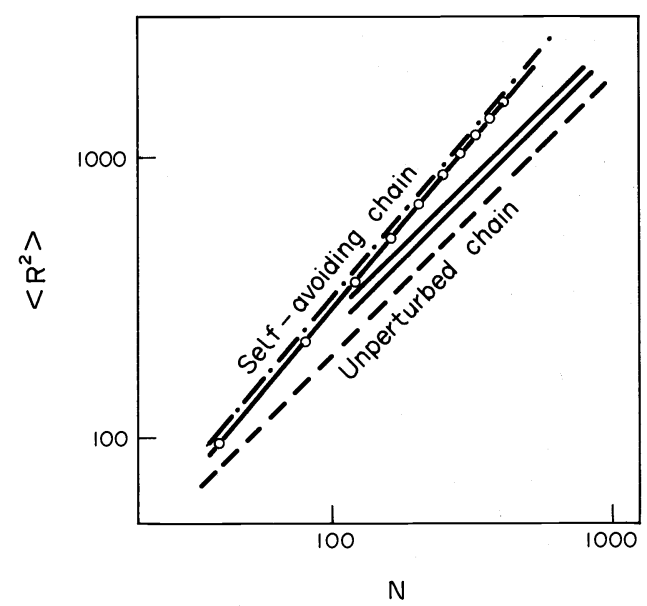

Fig. 2. Plots of mean-square end-to-end distance $\left\langle\mathrm{R}^{2}\right\rangle$ vs. chain size $\mathrm{N}$ for a "random" copolymer of equimolar composition on a tetrahedral lattice with a non-intersecting interaction only between unlike segments (circles). The dot-dash line is for a completely self-avoiding (homopolymer) chain on the same lattice, and the two solid lines are for the triblock (above) and diblock (below) equivalents on a simple cubic lattice. Reproduced from Ref. 21 by courtesy of American Chemical Society.

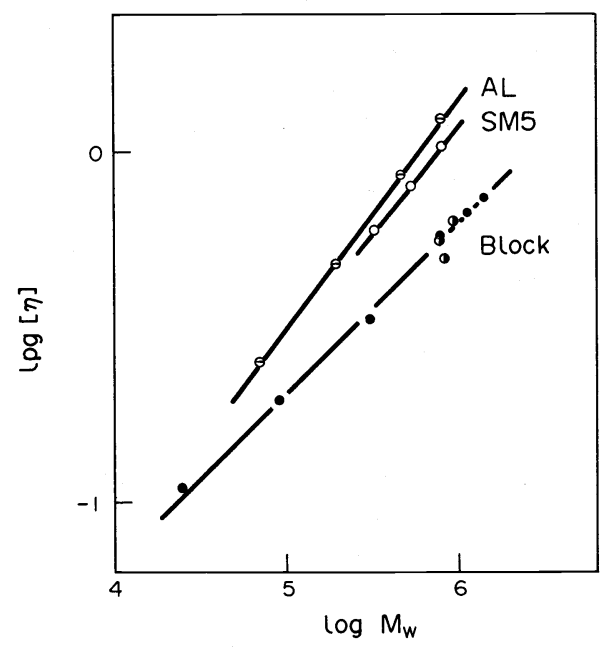

Fig. 3. Plots of $\log [\eta]$ vs. $\log M_{W}$ for S-M alternating (AL), S-M statistical(SM5), PS-PMMA diblock (half-filled circles) and PMMA-PS-PMMA triblock (filled circles) copolymers in cyclohexanol at $81^{\circ} \mathrm{C}$. Partly reproduced from Ref. 21 by courtesy of American Chemical Society.

a non-intersecting interaction only between unlike segments (a common theta state), and we tentatively identified a monomer unit as a segment. The chain architecture was assumed to be "random", the population of the A-B linkages as well as the average composition being equal to 0.50 . The behavior of the di- and triblock equivalents predicted from the data on the cubic lattice are also included in Fig. 2. The figure shows that the mean-square end-to-end distance $\left\langle\mathrm{R}^{2}\right\rangle$ of the random copolymer varies with $\mathrm{N}$ almost parallelly to that of the completely selfavoiding (homopolymer) chain simulated by Wall and Erpenbeck (29), giving the relation $\left\langle\mathrm{R}^{2}\right\rangle \propto \mathrm{N}^{\nu}$ with $\nu$ as large as 1.2. This means that in the presence of such a (strong) repulsive interaction between unlike segments, a random copolymer in a common theta solvent behaves much like a homopolymer chain in a good solvent but essentially differently from the block copolymers whose $\nu$ values are approximately unity as already pointed out.

In Fig. 3, we collected intrinsic viscosity data obtained for S-MMA alternating and statistical copolymers (Ref. 17) and PS-PMMA diblock (Ref. 22) and PMMA-PS-PMMA triblock (Ref. 11) copolymers in cyclohexanol at the temperature $81^{\circ} \mathrm{C}$, which approximately is a theta solvent for both PS and PMMA (Ref. 16). All these copolymers have nearly equimolar composition. The figure reveals that the Mark-Houwink exponent a is about 0.5 for the block copolymer, while it 
is much larger than 0.5 for the statistical $(a=0.62)$ and alternating $(a=0.65)$ copolymers. Even though such a comparison may be complicated by problems such as those described in the next section, these data do indicate that the Monte Carlo prediction is substantially correct.

In summary, due to their architectural features, block copolymers reflect only weakly the heterosegment interaction on their conformation. This becomes more and more true as the solvent power towards the parent polymers becomes better and the individual blocks get more and more expanded, thus decreasing the chance of the unlike segments to encounter (Ref. $18 \&$ $22)$. On the other hand, the statistical class of copolymers reflect the interaction more directly and strongly on their conformation. In the next section, we will examine the conformational properties of this class of copolymers in more detail.

\section{A NEW ANALYSIS OF VISCOMETRIC DATA ON COPOLYMERS}

Thus far, intrinsic viscosity data on copolymers have been customarily analyzed according to the two-parameter scheme (see, e.g., Ref. 30) established for homopolymers. The two parameters $A$ and $B$ characterizing the short-range and long-range interactions, respectively, thus deduced are generally functions of the composition and architecture of the copolymer. Aside from the functional forms of the parameters, such an approach would be justified, if and only if the conformation of the copolymer or the three-dimensional distribution of the segments is similar to that of a homopolymer. Apparently, statistical copolymers with a sufficient number of A-B linkages meet this requirement, but block copolymers do not in general.

The validity of the two-parameter scheme approach has been confirmed, to a certain extent, by experiments on statistical copolymers. Notably, Kotaka et al. ( $9 \& 17)$ have shown that the intrinsic viscosity [n] of statistical and alternating copolymers of $S$ and MMA becomes precisely proportional to the square-root of the molecular weight $M$ in solvents in which the second virial coefficient $A_{2}$ vanishes. This indicates that the "theta concept" is valid for this class of copolymers as well as for homopolymers. They furthermore analyzed the [ $\mathrm{n}$ ] data in good solvents according to the method of Stockmayer and Fixman (31) and confirmed that it provides reasonable estimates of the short-range parameter A. [There is, however, a report on S-MMA statistical copolymers, claiming that the parameter A thus estimated is dependent on solvent and temperature (Ref. 10)]. An important finding relevant to the short-range interaction in S-MMA copolymers is that the characteristic ratios of the copolymers are larger than the composition average of those of the parent polymers. The deviation was found to be approximately proportional to the population of S-MMA linkages or the "run number", namely, the alternating copolymer has a larger unperturbed dimension than the statistical copolymer with the same molecular weight and composition (Ref. 17).

Similar dependence was found also for the long-range interaction parameter $B$ estimated from the Stockmayer-Fixman plot. The result was interpreted by regarding dyads, rather than single monomeric units, as segments (Ref. 17). According to this treatment, the long-range interaction in a copolymer chain is expressed in terms of three parameters characterizing the interactions of solvent with polymer 1 , polymer 2 and 1-2 alternating copolymer, respectively. This treatment was successful to interpret the architectural dependence of the long-range interaction in individual solvents, but obviously it does not help to understand the observed dependence of the interaction parameter on solvent.

In Table 1, we collected the relevant data on S-MMA statistical (coded SM) and alternating (coded AL) copolymers (Ref. 17) along with those on the parent polymers (Ref. 22). The parameter $B$ was reevaluated according to the following semi-empirical relation between the hydrodynamic expansion factor $\alpha_{\eta}$ and the excluded volume parameter $z$,

$$
\alpha_{\eta}^{4}=1+(7 / 5) z
$$

or in terms of the parameters $A$ and $B$

$$
\left([n] / \mathrm{M}^{1 / 2}\right)^{4 / 3}=\mathrm{K}^{4 / 3}+0.462 \mathrm{~K}^{1 / 3} \Phi_{\mathrm{o}} \mathrm{BM}^{1 / 2}
$$

with $K=A^{3} \Phi_{0}$ and the value of the viscosity constant $\Phi_{0}$ of $2.68 \times 10^{23}$ due to Pyun and Fixman (32). It may be seen that Eq. (1) provides, for small as well as large values of $\mathrm{Z}$, a reasonable fit to the homopolymer data collected and analyzed by Yamakawa (30). Figure 4 shows an example of the plot indicated by Eq. (2). It seems that the plot is linear up to a considerably high molecular weight, thus providing less ambiguous estimates of $A$ and $B$ than, e.g., the Stockmayer-Fixman plot. If the two-parameter notations are brought into correspondence with those of the Flory-Huggins lattice theory (33), we have the solvent-polymer interaction parameter $X_{O P}$ listed in Table 1 ( the subscript $P$ denotes either homopolymers 1 and 2 or copolymer $C$ ). $\mathrm{XP}$ As to the $\chi$ parameter of a copolymer, Stockmayer et al. (3) have suggested the simple relation,

$$
x_{0 C}=\phi_{1} x_{01}+\phi_{2} x_{02}-\phi_{1} \phi_{2} x_{12}
$$




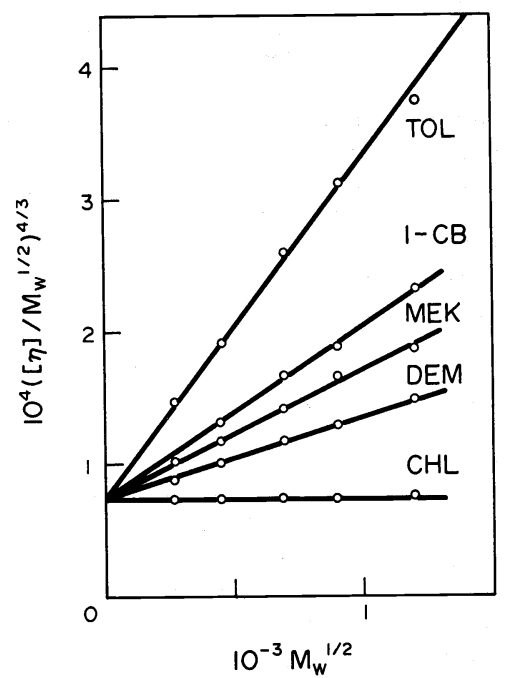

Fig. 4. Plots of $\left([\eta] / M_{W}^{1 / 2}\right)^{4 / 3}$ vs. $M_{W}^{1 / 2}$ for S-MMA alternating copolymers in toluene (TOL), 1-chlorobutane (1-CB), 2-butanone (MEK) and diethyl malonate (DEM) at $30^{\circ} \mathrm{C}$. The cyclohexanol (CHL) data are relevant to the theta temperature of the copolymer, $61^{\circ} \mathrm{C}$.

TABLE 1. Values of interaction parameters $B, X_{0 P}$ and $X_{12}$ for S-MMA copolymers and the parent homopolymers in various solvents at $30^{\circ} \mathrm{C}$ )

\begin{tabular}{lrrc}
\hline Solvent and polymer & $10^{27} \mathrm{~B}$ & $\mathrm{X}_{\mathrm{OP}}$ & $\mathrm{X}_{12}$ \\
\hline 1-chlorobutane $\left(\mathrm{V}_{0}=105.7, \delta_{0}=8.4\right)$ & & & \\
\hline PS & 1.12 & 0.4592 & - \\
PMMA & -0.09 & 0.5041 & - \\
AL & 1.14 & 0.4518 & 0.113 \\
SMA & 1.12 & 0.4534 & 0.104 \\
SM3 & 0.59 & 0.4741 & 0.070 \\
toluene $\left(\mathrm{V}_{0}=107.4, \delta_{0}=8.9\right)$ & & & \\
PS & 2.17 & 0.4197 & - \\
PMMA & 1.16 & 0.4449 & - \\
AL & 2.29 & 0.4017 & 0.119 \\
SMA & 2.09 & 0.4117 & 0.077 \\
SM3 & 1.75 & 0.4218 & 0.068 \\
2-butanone $\left(\mathrm{V}_{0}=90.8, \delta_{0}=9.3\right)$ & & & \\
PS & 0.54 & 0.4831 & - \\
PMMA & 1.04 & 0.4582 & - \\
AL & 0.86 & 0.4688 & 0.012 \\
SMA & 1.02 & 0.4636 & 0.035 \\
diethy1 malonate $\left(\mathrm{V}_{0}=153.3, \delta_{0}=9.5\right)$ & & & \\
PS & -0.07 & 0.5035 & - \\
PMMA & 1.08 & 0.4267 & - \\
AL & 0.53 & 0.4673 & 0.004 \\
SMA & 0.75 & 0.4551 & 0.060 \\
SM3 & 0.91 & 0.4420 & 0.044 \\
\hline
\end{tabular}

a $\mathrm{AL}$ : alternating copolymer, SMA: statistical copolymer $\left(\mathrm{m}_{1}=0.52\right.$ and $\mathrm{r}=0.67)$, SM3: statistical copolymer $\left(\mathrm{m}_{1}=0.29\right.$ and $\left.\mathrm{r}=0.51\right)$

where $\phi$ is the volume-composition of the copolymer (the authors defined Eq. (3) in terms of the mole-composition $\mathrm{m}$ instead of $\phi)$, and $\chi_{12}$ is the parameter characterizing the $1-2$ interaction. Values of $X_{12}$ computed from Eq. (3) are given in Table 1. As already implied, they vary from sample to sample and, in addition, they are strongly dependent on solvent. As yet, no theory is available that comprehensively accounts for these architectural and solvent dependences of $X_{12}$. 
Here we propose writing $\chi_{0 C}$ as a sum of the two terms

$$
x_{O C}=x_{O C}^{s}+x_{O C}{ }^{H}
$$

with the entropic term $\chi_{O C}{ }^{S}$ given as a composition average as

$$
x_{0 C}{ }^{s}=\phi_{1} x_{01}^{s}+\phi_{2} x_{02}^{s}
$$

and the enthalpic term $\chi_{O C}{ }^{H}$ expressed in terms of the solubility parameters $\delta_{C}$ and $\delta_{0}$ of the copolymer and solvent as

$$
\mathrm{X}_{\mathrm{OC}}^{\mathrm{H}}=\left(\mathrm{V}_{0} / \mathrm{RT}\right)\left(\delta_{\mathrm{C}}-\delta_{0}\right)^{2}
$$

In Eq. (6), $R$ is the gas constant, $T$ is the absolute temperature, and $V_{0}$ is the molar volume of the solvent. Then we assume that $\delta_{C}$ is given in the form

$$
\delta_{C}=\phi_{1} \delta_{1}+\phi_{2} \delta_{2}+r \Delta \delta
$$

where $\Delta \delta$ is a constant characteristic of a given pair of polymers. That is, the solubility parameter of a copolymer is assumed to differ from the composition average of those of the homopolymers by an amount proportional to the population $r$ of the 1-2 1inkages. This form of $\delta_{C}$ has been suggested in a previous work (Ref. 17). From Eq. (4) through (7), we have the following expression for the $\chi_{12}$ as defined by Eq. (3):

$$
\chi_{12}\left(\mathrm{RT} / \mathrm{V}_{0}\right)=\left(\delta_{2}-\delta_{1}\right)^{2}+\left(2 \mathrm{r} \Delta \delta / \phi_{1} \phi_{2}\right)\left[\delta_{0}-\left(\phi_{1} \delta_{1}+\phi_{2} \delta_{2}\right)-(\mathrm{r} \Delta \delta / 2)\right]
$$

Equation (8) suggests that for a given solvent, $X_{12}$ may be different for different $r$ and $\phi$, and for a given copolymer, the quantity $\chi_{12}\left(\mathrm{RT} / \mathrm{v}_{0}\right)$ may vary linearly with the solvent solubility parameter $\delta_{0}$. The parameter $\mathrm{r}$ may be determined experimentally or estimated according to the kinetics of copolymerization (Ref. 34).

The data presented in Table 1 were analyzed according to Eq. (8). Figures 5a-5c show the plot of $X_{12}\left(\mathrm{RT} / \mathrm{v}_{0}\right)$ vs. $\delta_{0}$ for the individual copolymers. The straight lines in the figures were calculated from Eq. (8) with $\delta_{1}=9.16, \delta_{2}-\delta_{1}=0.44$, and $\Delta \delta=-0.070$. These parameter

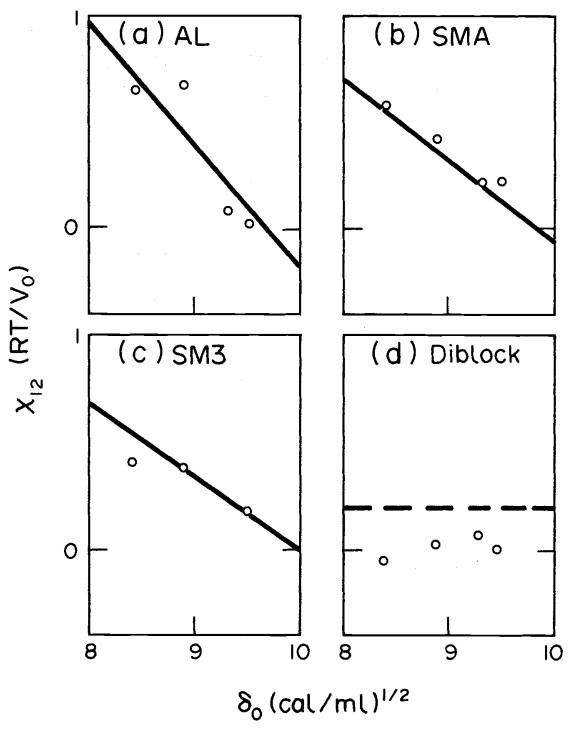

Fig. 5. Plots of $X_{12}\left(R T / V_{0}\right)$ vs. solvent solubility parameter $\delta_{0}$ for S-MMA alternating (a), S-MMA statistical (b \& c) and PS-PMMA diblock (d) copolymers in various solvents at $30^{\circ} \mathrm{C}$. The solid lines in $\mathrm{Fig}$. $5 \mathrm{a}-5 \mathrm{c}$ were calculated according to Eq. (8). The broken line in Fig. 5d is for the "ideal" chain described in the text.

values were determined by a least-squares calculation by giving all the data points the same significance. With possible errors in both $\chi_{12}$ and $\delta_{0}$ in mind, the agreement between the theory and experiment is good. Moreover, the values of $\delta_{1}, \delta_{2}$ and $\Delta \delta$ thus estimated seem quite reasonable. We hence concluded that the observed dependence of $\chi_{12}$ on both chain 
architecture and solvent originates from a rather trivial deviation of the copolymer solubility parameter from the composition average. More details of this analysis will be given elsewhere (Ref. 35).

If the squared difference $\left(\delta_{2}-\delta_{1}\right)^{2}$ estimated above is brought into correspondence with $\chi_{12}$ by the relation

$$
x_{12}^{\circ}=\left(\delta_{2}-\delta_{1}\right)^{2}\left(\mathrm{~V}_{0} / \mathrm{RT}\right)
$$

we have an estimate for the "true" interaction parameter $\chi_{12}{ }^{\circ}$ between PS and PMMA. If we take the value $100 \mathrm{ml}$ as a reference molar volume of solvent (Ref. 36), this gives $\chi_{12} 0=0.03$. Discussion on this estimate will be given in the next section.

The arguments given thus far imply that it might be possible to extract from copolymer data the interaction parameter between the parent polymers. One method is studying a sufficient number of copolymers differing in $\mathrm{r}$ and/or $\phi$, as we have done above. Alternatively, one can measure a single copolymer in which (i) the average runs of either monomeric units are much shorter than the total length of the chain but (ii) they are still long enough that the alternating linkages do not figure significantly (Ref. $3 \& 17$ ). As has been pointed out, block copolymers of lower architectural orders (e.g., di- and triblock chains) meet the second, but not the first requirement. Tentatively, we have analyzed the viscosity data of PS-PMMA diblock samples in a similar way as we treated the SM and AL copolymers. Figure $4 \mathrm{~d}$ shows the result. Each point in the figure represents an average over many samples differing in composition. Quantitatively, such an analysis may be questionable in several respects. These include problems regarding universality of the viscosity constant, sample heterogeneity in both molecular weight and composition (Ref. 22) and micellization in selective solvents (Ref. $6,37-43)$. Nevertheless, the figure strongly suggests that the apparent interaction parameter of the block copolymer is nearly zero, independent of the solvents. This is in line with what we have described in the preceding section.

\section{LIGHT SCATTERING FROM POLYMER/POLYMER/SOLVENT TERNARY SOLUTIONS}

Another possible route to characterization of polymer-polymer interactions is provided by light scattering on ternary solutions. In the past, there were several interesting studies of this kind (Ref. 44-51). However, it has been recognized that the light scattering method, especially when applied to dilute ternary solutions, has a serious defect: it lacks precision. The effects of polymer-solvent interactions are usually so large that they are liable to mask the relatively small contribution from polymer-polymer interaction. Recently, we proposed a method which may overcome the defect and simplify experiments (Ref. 52). It is based on the use of a solvent in which the refractive index increments of the two polymers are opposite in sign so that the scattering components relevant to the polymer-solvent interactions cancel each other. It is thus possible to observe the polymer-polymer interaction selectively. In what follows, we briefly describe the principle of the method along with preliminary results obtained by applying it to PS/PMMA/bromobenzene solutions (Ref. 53-55).

We combine the light scattering theory due to Zernicke and others (56-58) with the FloryHuggins expression for the free-energy of mixing (Ref. 33) to have the following formula which is valid for low concentrations of the polymers (see, e.g., Ref. 44):

$$
\begin{aligned}
& \mathrm{R}_{0} / \mathrm{K} * \phi=\mathrm{B}_{0}+\mathrm{B}_{1} \phi+0\left(\phi^{2}\right) \\
& \mathrm{B}_{0}=\psi_{1}{ }^{2} \mathrm{r}_{1} \mathrm{x}_{1}+\psi_{2}{ }^{2} \mathrm{r}_{2} \mathrm{x}_{2} \\
& \mathrm{~B}_{1}=-\left(\psi_{1} \mathrm{r}_{1} \mathrm{x}_{1}+\psi_{2} \mathrm{r}_{2} \mathrm{x}_{2}\right)\left[\psi_{1} \mathrm{r}_{1} \mathrm{x}_{1}\left(1-2 \mathrm{x}_{01}\right)+\psi_{2} \mathrm{r}_{2} \mathrm{x}_{2}\left(1-2 \mathrm{x}_{02}\right)\right]-2 \psi_{1} \psi_{2} \mathrm{r}_{1} \mathrm{r}_{2} \mathrm{x}_{1} \mathrm{x}_{2} \mathrm{x}_{12}
\end{aligned}
$$

In these equations, $R_{0}$ is the forward scattering intensity, $K^{*}$ is a constant, $r_{j}$ is the weight average (see Ref. 59) degree of chain length, $\psi_{i}$ is the refractive index increment $\partial n / \partial \phi_{i}, \phi_{i}$ is the volume fraction of polymer $i, \phi=\phi_{1}+\phi_{2}$, and $x_{i}=\phi_{i} / \phi$. We now consider the condition

$$
\psi_{1} r_{1} x_{1}+\psi_{2} r_{2} x_{2}=0
$$

which transforms Eq. (8)-(10) to

$$
\mathrm{K} * \phi / \mathrm{R}_{0}=\mathrm{B}_{0}^{-1}+\left(2 \psi_{1} \psi_{2} \mathrm{r}_{1} \mathrm{r}_{2} \mathrm{x}_{1} \mathrm{x}_{2} \mathrm{~B}_{0}^{-2}\right) \mathrm{X}_{12} \phi+0\left(\phi^{2}\right)
$$

Clearly, then, the initial slope of the $\phi / R_{0}$ vs. $\phi$ plot is directly proportional to $\chi_{12}$, regardless of the thermodynamic properties of the solvent. This is the greatest advantage of this method. Another advantage becomes evident when we deal with a symmetrical system, a system in which $x_{01}=x_{02}, r_{1}=r_{2}$, and $x_{1}=x_{2}$ (but $\psi_{1}=-\psi_{2}$ ). Examining the light scattering 
equation in a non-expanded form (see, e.g., Ref. 58), we see that all the terms in Eq. (12) equal to or higher than the second power of $\phi$ simultaneously vanish for a symmetrical system. Provided that $X_{12}$ is independent of concentrations, the $\phi / R_{0}$ vs. $\phi$ plot is, thus, linear up to the spinodal at which $R_{0}$ becomes "infinitely" large. For this reason, we can expect to determine $X_{12}$ with high precision, even when it is very small.

PS/PMMA/bromobenzene dilute solutions

Figure 6 shows the plot of $\mathrm{c} / \mathrm{R}_{0}$ vs. $\mathrm{c}$ for a PS/PMMA blend in bromobenzene at $30^{\circ} \mathrm{C}$, where $\mathrm{c}$ is the total polymer concentration in $\mathrm{g} / \mathrm{ml}$. The blending ratio $\mathrm{x}$ of the two polymers was so determined as to satisfy the condition in Eq. (11). The forward intensity $\mathrm{R}_{0}$ was determined

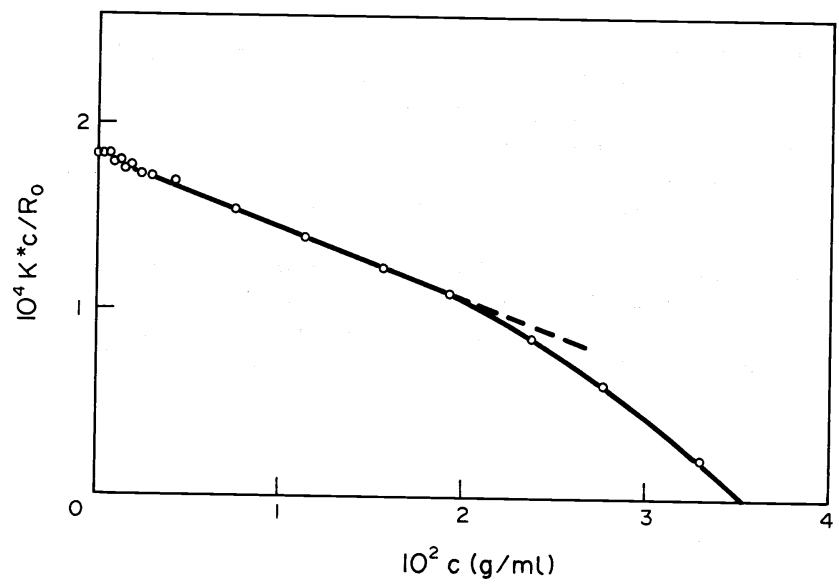

Fig. 6. Plot of $\mathrm{K} * \mathrm{c} / \mathrm{R}_{0}$ vs. $\mathrm{c}$ for a PS $\left(\mathrm{M}_{\mathrm{w}}=2.42 \times 10^{6}\right) / \mathrm{PMMA}\left(2.19 \times 10^{6}\right)$ blend in bromobenzene at $30^{\circ} \mathrm{C}$.

according to the conventional extrapolation method. The figure exhibits a rather small, negative initial slope, from which it is immediately clear that the system is characterized by a small, positive value of $\chi_{12}$, since $\psi_{1} \psi_{2}$ is negative in our systems. We also note that the curve is linear up to a considerably high concentration. It was thus possible to determine $X_{12}$ with little ambiguity, despite its small magnitude. The value of $\chi_{12}$ evaluated from the initial slope is designated as $\chi_{12}{ }^{d i l}$ and given in Table 2 along with the results
for several other blends.

TABLE 2. Values of $X_{12}$ and $\phi *$ for PS/PMMA/bromobenzene systems at $30^{\circ} \mathrm{C}$

\begin{tabular}{|c|c|c|c|c|c|}
\hline \multicolumn{2}{|c|}{$10^{5} \mathrm{M}_{\mathrm{w}}$ a) } & \multirow{2}{*}{$\xi_{1}$ b) } & \multirow{2}{*}{$x_{12}{ }^{\mathrm{di1}}$} & \multirow{2}{*}{$\left.x_{12}{ }^{\circ} \mathrm{c}\right)$} & \multirow{2}{*}{$\phi^{*}$} \\
\hline PS & PMMA & & & & \\
\hline 24.2 & 21.9 & 0.455 & 0.0026 & 0.024 & 0.019 \\
\hline 7.75 & 6.27 & 0.427 & 0.0030 & 0.022 & 0.023 \\
\hline 2.83 & 2.11 & 0.407 & 0.0040 & 0.021 & 0.050 \\
\hline 0.25 & 0.25 & 0.487 & 0.0117 & 0.031 & 0.180 \\
\hline
\end{tabular}

Table 2 clearly indicates that $\chi_{12}{ }^{\text {dil }}$ is molecular weight dependent: the larger the molecular weights are, the smaller $x_{12}$ dil is. The trend is similar to that of the second virial coefficient $A_{2}$ in a binary solution. Thus it was tempting to examine this matter in light of the $\mathrm{A}_{2}$ theory. According to the theory, $\chi_{12}{ }^{\text {dil }}$ should be approximately related to $\chi_{12}{ }^{\circ}$, i.e., the "true" $x_{12}$ by

$$
\chi_{12}^{\mathrm{dil}}=(\mathrm{d} \Psi / \mathrm{d} \overline{\mathrm{Z}}) \mathrm{X}_{12}{ }^{\circ}
$$

where $\Psi$ is the penetration factor, which is a function only of $\bar{z}$, the excluded volume 
parameter divided by the cubic expansion factor $\alpha^{3}$. Equation (13) is correct for a symmetrical system with a small $X_{12}{ }^{\circ}$. Independent studies on the binary solutions have revealed that the present systems approximately satisfy the symmetry conditions (Ref. 54). Based on the Kurata-Yamakawa theory on $\Psi(60)$ and the Yamakawa-Tanaka theory on $\alpha(61)$, we estimated the value of $x_{12}{ }^{\circ}$ for the individual blends (Table 2). They no longer seem to be molecular weight dependent, revealing that the observed trend of $\chi_{12}$ is a real phenomenon originating from an excluded volume effect. This dilute solution study suggests that $\chi_{12}{ }^{\circ}$ between PS and PMMA is about 0.025 for the bromobenzene molar volume, $106 \mathrm{ml}$.

\section{PS/PMMA/bromobenzene solutions of finite concentration}

At present, a rigorous analysis of light scattering data on ternary solutions with a finite concentration is difficult to make, since the scattering intensity depends on the subtle details of still-unknown composition dependence of the interaction parameters $X_{0 i}$ and $X_{12}$, as will be discussed elsewhere (Ref. 55). As a first step to this problem, one could assume that $X$ 's are dependent only on $\phi$, the total polymer concentration. This makes the analysis feasible, since $X_{0 i}$ 's may, then, be determined from independent studies on the binary systems. If, in addition, the ternary system considered is symmetrical, the analysis is further simplified: It may be easily shown that the scattering function is independent of $\chi_{01}$, so far as the symmetry conditions are met at all concentrations.

The finite concentration data on the above ternary solutions were analyzed under the assumption that $X_{0 i}$ 's are independent of $\phi$, which is a good approximation for these nearly symmetrical systems. A typical example showing the concentration dependence of the $\chi_{12}$ thus estimated is presented in Fig. 7. In this analysis, the composition dependence of solution

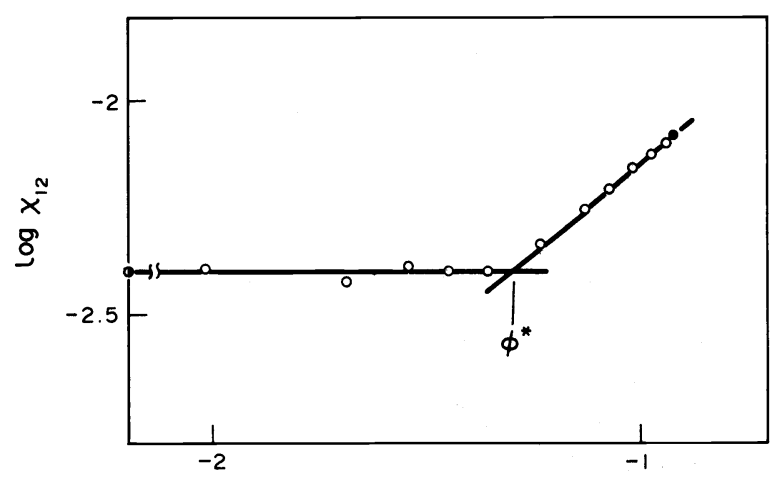

$\log \phi$

Fig. 7. Plot of $\log X_{12}$ vs. $\log \phi$ for a PS $\left(M_{\mathrm{w}}=2.83 \times 10^{5}\right) / \mathrm{PMMA}\left(2.11 \times 10^{5}\right)$ blend in bromobenzene at $30^{\circ} \mathrm{C}$. The filled and half-filled circles refer to the spinodal and the dilute limit, respectively.

density and refractivity was adequately taken into account. Interestingly, the figure implies that there exists a characteristic concentration $\phi *$ below which $X_{12}$ is approximately constant and equal to $X_{12}$ dil but above which it increases sharply with increasing $\phi$. This suggests a rather drastic change in the degree of interpenetration of the polymer coils, and brings to mind the recent idea of the "dilute-to-semi-dilute crossover" (Ref. 62-64). Numerically, the concentration $\phi^{*}$ was found to be closely related to the "overlap concentration" given by $\mathrm{v}_{\mathrm{w}} /\left\langle\mathrm{s}^{2}\right\rangle_{\mathrm{w}}{ }^{3 / 2}$, where $\mathrm{v}_{\mathrm{w}}$ and $\left\langle\mathrm{s}^{2}\right\rangle_{\mathrm{w}}{ }^{1 / 2}$ are the weight-average molecular volume and radius of gyration of the polymers. According to de Gennes (64), $\chi_{12}$ in a semi-dilute solution depends on concentration but not on molecular weight. In Fig. 8, the $X_{12}$ data relevant to concentrations above $\phi^{*}$ are collected and plotted against $\phi$. Apparently, the data points form a single composite curve.

The absence of molecular weight dependence disclosed by the above study may be of special significance. In particular, it justifies, at least in a certain aspect, trials for estimating the $x_{12}$ in a dry blend on the basis of concentrated-solution data. At present, no adequate theory is available which explains the functional form of $\chi_{12}$ as displayed in Fig. 8 and permits prediction of its behavior at concentrations closer to $\phi=1$. However, the curve in Fig. 8 suggests that $X_{12}$ converges to 0.03 or thereabout at $\phi=1$.

This value is in favorable agreement with the dilute solution value corrected for the excluded volume effect and also with the copolymer value given in the preceding section. We also note that the conventional solubility parameter approach [cf. Eq. (7)] gives an estimate of $\chi_{12}$ at least compatible with these results. In fact, values of $\delta_{P S}$ and $\delta_{P M M A}$ reported in the 


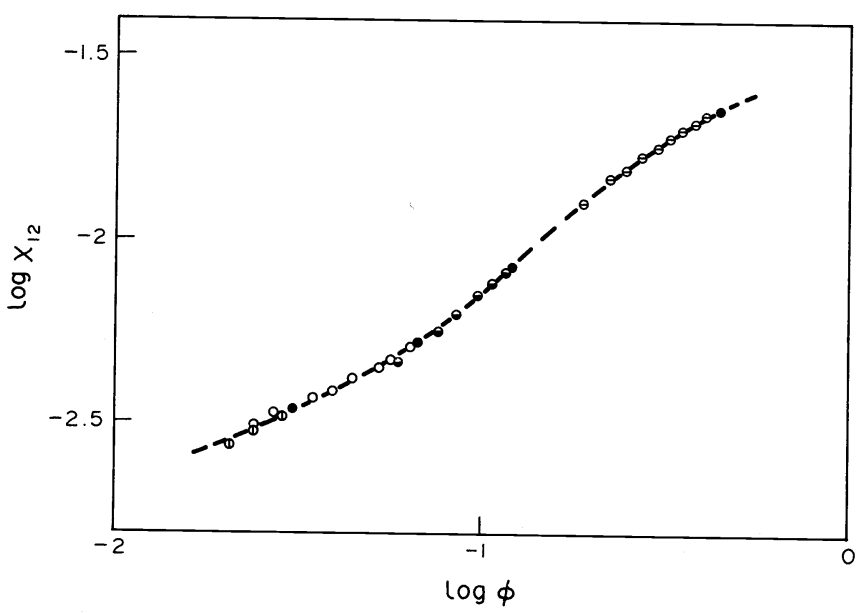

Fig. 8. Plot of $\log X_{12}$ vs. $\log \phi$ for PS/PMMA blends in bromobenzene at $30^{\circ} \mathrm{C}$, relevant to concentrations above $\phi^{*}$. Different symbols refer to different blends (the filled circle represents the spinodal for each blend).

literature (65) are considerably scattered, but the two groups of values are rather close to each other. Accordingly, the precision cannot be high. The value of $x_{12}$ given in Table 3 is based on the parameter values $\delta_{\mathrm{PS}}=9.1$ and $\delta_{\mathrm{PMMA}}=9.5$ obtained by the same authors

according to the same method (Ref. 66). In any case, the fair agreement among the values

TABLE 3. Values of $X_{12}^{\circ}$ between PS and PMMA estimated by various methods a)

\begin{tabular}{ll}
\hline Method & $x_{12}{ }^{\circ}$ \\
\hline copolymer intrinsic viscosity & $0.03_{2}$ \\
dilute ternary solution (average) & $0.02_{4}$ \\
concentrated ternary solution & $0.03_{0}$ \\
solubility parameter (Ref. 66) & $0.02_{7}$ \\
\hline
\end{tabular}

${ }^{a}$ Value for a reference solvent volume of $100 \mathrm{ml} / \mathrm{mol}$ and for a temperature around $30^{\circ} \mathrm{C}$.

estimated by the different methods indicates that solution data could be used to provide some quantitative information on polymer-polymer interaction. However, one should not hasten to generalize this indication obtained for the particular systems: For example, the behavior of a ternary solution composed of an asymmetrical pair of polymers is as yet understood very little (see, e.g., Ref. 59).

Acknowledgement - This work was supported in part by a Grant-in-Aid for Scientific Research, the Ministry of Education, Japan (56470081).

\section{REFERENCES}

1. A. Noshay and J.E. McGrath, Block Copolymers. Overview and Critical Survey, Academic Press, New York (1977).

2. D.C. Allport and W.H. Jones, ed., Block Copolymers, Willey, New York (1973).

3. W.H. Stockmayer, L.D. Moore, Jr., M. Fixman, and B.N. Epstein, J. Polym. Sci., 16, 517 (1955).

4. G.M. Burnett, P. Mears, and C. Paton, Trans. Faraday Soc., 58, 737 (1962).

5. H. Utiyama, Dessertation, Kyoto University (1963).

6. S. Krause, J. Phys. Chem., 68, 1948 (1964).

7. H. Benoit, Ber. Bunsenges. Physik. Chem., 70, 286 (1966).

8. L.A. Utracki, R. Simha, and L.J. Fetters, J. Polym. Sci., Part A2, 6, 2051 (1968). 
9. T. Kotaka, Y. Murakami, and H. Inagaki, J. Phys. Chem., 72, 829 (1968).

10. A. Dondos and H. Benoit, Makromol. Chem., 118, 165 (1968).

11. H. Ohnuma, T. Kotaka, and H. Inagaki, Polymer, 10, 501 (1969): T. Kotaka, H. Ohnuma, and H. Inagaki, Polymer, 10, 517 (1969).

12. J. Prud'homme and S. Bywater, Macromolecules, 4, 543 (1971).

13. H. Utiyama, K. Takenaka, M. Mizumori, and M. Fukuda, Macromolecules, 7, 28 (1974).

14. T. Tanaka-Fukuda, T. Kotaka, and H. Inagaki, Macromolecules, $\underline{7}, 311$ (1974).

15. M. Shima, E. Ogawa, and K. Konishi, Makromo1. Chem., 177, 241 (1976).

16. T. Kotaka, H. Ohnuma, and Y. Murakami, J. Phys. Chem., 70, 4099 (1966).

17. T. Kotaka, T. Tanaka-Fukuda, H. Ohnuma, Y. Murakami, and H. Inagaki, Polymer J., 1,245 (1971).

18. T. Tanaka-Fukuda, T. Kotaka, and H. Inagaki, Macromolecules, 9, 561 (1976).

19. T.M. Birstein, A.M. Skvortsov, and A.A. Sariban, Macromolecules, 9, 888 (1976).

20. J. Bendler, K. Solc, and W. Gobush, Macromolecules, 10, 635 (1977).

21. T. Tanaka-Fukuda, T. Kotaka, K. Ban, M. Hattori, and H. Inagaki, Macromolecules, 10,960 (1977).

22. T. Tanaka-Fukuda, M. Omoto, and H. Inagaki, J. Macromol. Sci.-Phys., B17, 229 (1980).

23. T. Tanaka-Fukuda, M. Omoto, and H. Inagaki, Macromolecules, $12,146(1979)$.

24. A. Dondos, P. Rempp, and H. Benoit, Makromol. Chem., 130, 233 (1969).

25. J.R. Urwin and M. Girolamo, Makromol. Chem., 150, 179 (1971).

26. A. Dondos, J. Polym. Sci., Part B, 9, 871 (1971).

27. J.P. Plante, N. Ho-Duc, and J. Prud'homme, Eur. Polym. J., 9, 77 (1973).

28. C.C. Han and B. Mozer, Macromolecules, 10, 44 (1977).

29. F.T. Wa11 and J.J. Erpenbeck, J. Chem. Phys., 30, 634, 637 (1959).

30. H. Yamakawa, Modern Theory of Polymer Solutions, Harper \& Row, New York (1971).

31. W.H. Stockmayer and M. Fixman, J. Polym. Sci., Part C, 1, 137 (1963).

32. C.W. Pyun and M. Fixman, J. Chem. Phys., 42, 3838 (1965).

33. P.J. Flory, Principles of Polymer Chemistry, Cornell University Press, Ithaca, N. Y. (1953).

34. H.J. Harwood and W.M. Ritchey, J. Polym. Sci., Part B, 2, 601 (1964).

35. T. Tanaka-Fukuda and H. Inagaki, to appear in Polymer J., 1984.

36. S. Krause, J. Macromo1. Sci. - Revs. Macromo1. Chem., C7, 251 (1972).

37. F.M. Merret, J. Polym. Sci., 24, 467 (1957).

38. T. Kotaka, T. Tanaka-Fukuda, and H. Inagaki, Polymer J., 3, 327 (1972).

39. J. Periard and G. Riess, Eur. Polym. J., $9,6 \overline{687(1973)}$.

40. C. Price and D. Woods, Eur. Polym. J., 9, 827 (1973).

41. H. Utiyama, K. Takenaka, M. Mizumori, M. Fukuda, Y. Tsunashima, and M. Kurata, Macromolecules, ㄱ, 515 (1974).

42. T. Kotaka, T. Tanaka-Fukuda, M. Hattori, and H. Inagaki, Macromolecules, 11, 138 (1978).

43. For a review, see, Z. Tuzar and P. Kratochvil, Adv. Colloid Interf. Sci., $\underline{6}, 201$ (1976).

44. W.H. Stockmayer and H.E. Stanley, J. Chem. Phys., 18, 153 (1950).

45. A.J. Hyde and A.G. Tanner, J. Colloid Interf. Sci., 28, 179 (1968).

46. R. Kuhn, H.-J. Cantow, and W. Burchard, Angew. Makromol. Chem., 2, 146, 157 (1968).

47. R. Kuhn and H.-J. Cantow, Makromol. Chem., 122, 65 (1969).

48. P. Kratochvil, J. Vorliciek, D. Strakova, and Z. Tuzar, J. Polym. Sci., Polym. Phys. Ed., 13,2321 (1975).

49. P. Kratochvil, D. Strakova, and Z. Tuzar, Br. Polym. J., 9, 217 (1977).

50. M.W.J. van den Esker and A. Vrij, J. Polym. Sci., Polym. Phys. Ed., 14, 1943 (1976).

51. M.W.J. van den Esker, J. Laven, A. Broekman, and A. Vrij, J. Polym. Sci., Polym. Phys. Ed., 14, 1953 (1976).

52. T. Tanaka-Fukuda and H. Inagaki, Macromloecules, 12, 1229 (1979).

53. T. Tanaka-Fukuda and H. Inagaki, Ann. Rep. Res. Inst. Chem. Fibers, Jpn., 39, 13 (1982).

54. T. Tanaka-Fukuda and H. Inagaki, to appear in Macromolecules, 1984.

55. T. Tanaka-Fukuda, M. Nagata, and H. Inagaki, to appear in Macromolecules, 1984.

56. F. Zernike, Arch. Neerland, 3A, 4, 74 (1917).

57. J.G. Kirkwood and R. Goldberg, J. Chem. Phys., 18, 54 (1950).

58. W.H. Stockmayer, J. Chem. Phys., 18, 58 (1950).

59. R. Konningsveld, H.A.G. Chirmin, and M. Gordon, Trans. Faraday Soc., A319, 331 (1970).

60. (a) M. Kurata, H. Fukatsu, H. Sotobayashi, and H. Yamakawa, J. Chem. Phys., 41, 139

(1964): (b) H. Yamakawa, J. Chem. Phys., 48, 2103 (1968).

61. H. Yamakawa and G. Tanaka, J. Chem. Phys., 47, 3991 (1967).

62. S.F. Edwards, Proc. Phys. Soc., 85,613 (1965); 88,265 (1966).

63. M. Daoud and G. Jannink, J. Phys (Paris), 37, 973 (1976).

64. P.G. de Gennes, Scalling Concepts in Polymer Physics, Corne11 University Press, Ithaca, N. Y. (1979).

65. H. Burrell, Solubility Parameter Values, in Polymer Handbook, J. Brandrup and E.H. Immergut, ed., Wiley, New York (1975).

66. D. Mangaraj, S.K. Bhatnagar, and S.B. Rath, Makromol Chem., 67, 75 (1963): D. Mangaraj, S. Patra, and S. Rashid, Makromo1. Chem. 65, 39 (1963) 